Variable thermal resistor based on self-powered Peltier effect

This article has been downloaded from IOPscience. Please scroll down to see the full text article.

2008 J. Phys. D: Appl. Phys. 41222001

(http://iopscience.iop.org/0022-3727/41/22/222001)

View the table of contents for this issue, or go to the journal homepage for more

Download details:

IP Address: 131.251.133.28

The article was downloaded on 02/04/2012 at 10:15

Please note that terms and conditions apply. 


\title{
Variable thermal resistor based on self-powered Peltier effect
}

\author{
Gao $\operatorname{Min}^{1}$ and N Md Yatim ${ }^{1,2}$ \\ ${ }^{1}$ School of Engineering, Cardiff University, Cardiff, CF24 3AA, UK \\ ${ }^{2}$ Islamic Science University of Malaysia, 71800 Nilai, Negeri Sembilan, Malaysia
}

Received 10 September 2008

Published 23 October 2008

Online at stacks.iop.org/JPhysD/41/222001

\begin{abstract}
Heat flow through a thermoelectric material or device can be varied by an electrical resistor connected in parallel to it. This phenomenon is exploited to design a novel thermal component-variable thermal resistor. The theoretical background to this novel application is provided and an experimental result to demonstrate its feasibility is reported.
\end{abstract}

Thermoelectric phenomena, which describe solid-state energy conversion processes between heat and electricity, are the basis for well-established applications such as power generation [1], refrigeration [2] and temperature sensing (thermocouples and infrared detectors) [3,4]. Other proposed applications of using thermoelectrics include vacuum gauge [5], signal converter [6] and dual-purpose heat pipe/converter [7]. Recently, the use of a thermoelectric module to realize an electrically controlled thermal resistance has been investigated by Szekely et al [8]. In their study, the variation in heat flow is achieved by changing the electric current supplied to the thermoelectric module. An external power source is required.

In this paper, we propose a novel configuration to realize variable thermal resistance without the need for an external power source. Electrical power generated due to the Seebeck effect is fed back to the thermoelectric material via an electrical resistor to produce a self-powered Peltier effect. This will result in an increase in heat flow through the thermoelectric material and consequently a decrease in temperature difference across it. The degree of change in heat flow or temperature difference can be controlled by the magnitude of electrical resistance. The principle of operation can be formulated based on thermoelectric device theory [2].

The proposed configuration is shown in figure $1(a)$. A thermoelectric material is sandwiched between two thermal bodies $\mathrm{A}$ and $\mathrm{B}$ of different temperatures $T_{\mathrm{A}}$ and $T_{\mathrm{B}}$. The two ends of the thermoelectric material are connected electrically to an electrical resistor, $R_{\mathrm{L}}$. Its equivalent thermoelectric circuit is shown in figure $1(b)$. Based on the Seebeck effect, the thermoelectric material under a temperature difference
$\Delta T=T_{\mathrm{A}}-T_{\mathrm{B}}$ serves as a power supply which has an electromotive force $\alpha \Delta T$ and internal resistance of $R_{\mathrm{i}}=$ $\rho(l / A)$, where $\alpha$ is the Seebeck coefficient, $\rho$ is the electrical resistivity and $l$ and $A$ are the length and cross-sectional area of the thermoelectric material, respectively.

The heat flow through the thermoelectric material depends on its thermal properties and operating conditions of the thermoelectric circuit. If the circuit is an open circuit (i.e. $R_{\mathrm{L}}=\infty$ ), the heat flow through the thermoelectric material is governed by the Fourier law,

$$
\begin{aligned}
\dot{Q}_{\mathrm{o}} & =\lambda \frac{A}{l}\left(T_{\mathrm{A}}-T_{\mathrm{B}}\right) \\
& =K \Delta T_{\mathrm{o}},
\end{aligned}
$$

where $\lambda$ is the thermal conductivity and $K=\lambda(A / l)$ is the thermal conductance of thermoelectric material. The subscript 'o' represents the thermoelectric circuit operating under the open-circuit condition.

In a closed-circuit condition (i.e. when $R_{\mathrm{L}}$ is finite), the voltage generated across thermoelectric material due to the Seebeck effect will result in an electric current that circulates through the thermoelectric material and external resistor,

$$
I=\frac{\alpha \Delta T_{\mathrm{c}}}{R_{\mathrm{i}}+R_{\mathrm{L}}},
$$

where $\Delta T_{\mathrm{c}}$ is the temperature difference between thermal bodies $A$ and $B$ under the closed-circuit condition. In general, $\Delta T_{\mathrm{c}}$ differs from $\Delta T_{\mathrm{o}}$ due to an additional heat flow caused by the Peltier effect when there is an electric current 


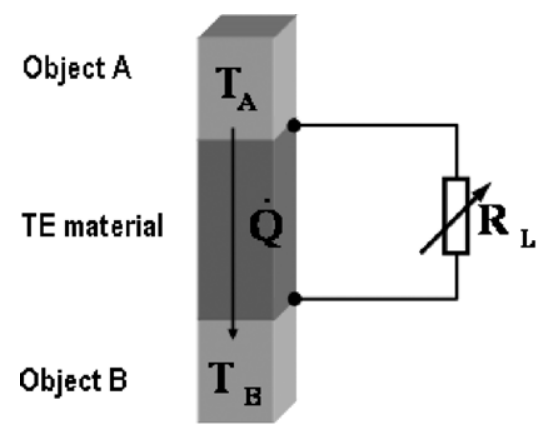

(a)

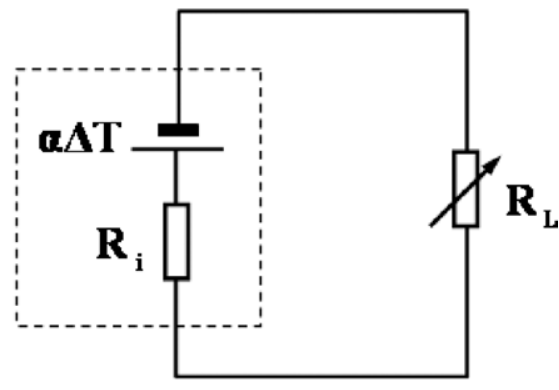

(b)

Figure 1. Principle of variable thermal resistor. (a) Thermoelectric material sandwiched between two thermal bodies and connected to a variable electrical resistor and $(b)$ equivalent thermoelectric circuit for the structure shown in $(a)$.

flowing through the thermoelectric material. Under such circumstances, the total heat flow through the thermoelectric material is given by [2]

$$
\dot{Q}_{\mathrm{c}}=\alpha T_{\mathrm{A}} I-\frac{1}{2} I R_{\mathrm{i}}^{2}+K \Delta T_{\mathrm{c}} .
$$

Substituting $I$ in equation (3) with the right-hand term of equation (2), and letting $T_{\mathrm{M}}=\left[(2 a-1) T_{\mathrm{A}}+T_{\mathrm{B}}\right] / 2 a^{2}$ with $a=1+R_{\mathrm{L}} / R_{\mathrm{i}}$, we obtain

$$
\dot{Q}_{\mathrm{c}}=\left(1+Z T_{\mathrm{M}}\right) K \Delta T_{\mathrm{c}},
$$

where $Z=\alpha^{2} /\left(R_{\mathrm{i}} K\right)$ is the thermoelectric figure of merit and $T_{\mathrm{M}}$ is a parameter associated with the temperatures of two thermal bodies $A$ and $B$ (under the open-circuit condition) and the ratio of the load to internal resistor, $R_{\mathrm{L}} / R_{\mathrm{i}}$.

It can be seen that equation (4) exhibits a similar form to equation (1) except for having an additional factor of $\left(1+Z T_{\mathrm{M}}\right)$, which depends on the thermoelectric figure of merit and the electrical resistance of internal and external resistors. This indicates that the relationship between heat flow and temperature difference in the proposed configuration obeys essentially the Fourier's law except that the effective thermal conductivity of the materials can be varied by an external electrical resistor. This forms the basis of a variable thermal resistor, which may be employed in various applications where the temperature difference between two thermal bodies needs to be controlled automatically, such as in package thermal measurements [8].

The 'proof of principle' of this proposed component was carried out using a thermoelectric module. It is noted that equation (4) is also valid for a thermoelectric module which consists of a large number of n- and p-type thermoelements connected electrically in series and thermally in parallel [9]. In this case, the total internal electrical resistance of a module is $R_{\mathrm{i}}=2 N \rho(l / A)$ and total thermal conductance is $K=$ $2 N \lambda(A / l)$, where $N$ is the number of the thermocouples in a module. A commercial module which has 127 pairs of thermocouples made from $\mathrm{Bi}_{2} \mathrm{Te}_{3}$ based alloys was used. The 'effective thermoelectric figure of merit' for the module is approximately $2.0 \times 10^{-3} 1 / \mathrm{K}$ at room temperature ${ }^{3}$. The

\footnotetext{
3 The 'effective' indicates that the thermoelectric figure of merit is determined from measuring performances of thermoelectric modules which include the adverse contributions from electrical and thermal contact resistances.
}

length and cross-sectional area of thermoelements are $1.7 \mathrm{~mm}$ and $1.4 \times 1.4 \mathrm{~mm}^{2}$, respectively. This gives an internal electrical resistance of $2.22 \Omega$ and thermal conductance of $0.44 \mathrm{~W} \mathrm{~K}^{-1}$.

The experiment was performed under a constant heat flow of $\dot{Q}=39 \mathrm{~W}$ with the cold side temperature of the module maintained at $T_{\mathrm{B}}=294 \mathrm{~K}$. The constant heat flow is provided by a heater assembly that has one surface in contact with the module surface using thermal paste to ensure good thermal contact. To minimize the heat leak to the surroundings, all other surfaces of the heater assembly are enclosed in a plastic box filled with a thick layer of insulting wools. The heat flux is monitored using two thermocouples inserted in a copper block, which is placed between heating element and module. The average deviation in $\dot{Q}$ is about $2.5 \%$. Under the opencircuit operation, the hot side temperature of the module is $T_{\mathrm{A}}=385 \mathrm{~K}$. This corresponds to a temperature difference of $\Delta T_{\mathrm{o}}=91 \mathrm{~K}$ across the module. However, in a closedcircuit operation, the temperature difference, $\Delta T_{\mathrm{c}}$, will change with the electrical resistance, $R_{\mathrm{L}}$. The result is displayed in figure 2. The solid circles represent the data obtained from experiment for different resistance values of $R_{\mathrm{L}}$. The dashed lines represent the calculated results using equation (4) for different $Z$ values. It can be seen that a maximum variation of about $37 \mathrm{~K}$ has been obtained using a commercial module by changing $R_{\mathrm{L}}$ from 0.01 to $222 \Omega$. The result is in a good agreement with theoretical calculation. Furthermore, figure 2 shows that the magnitude of reduction in $\Delta T_{\mathrm{c}}$ is proportional to the thermoelectric figure of merit. A much larger $\Delta T_{\mathrm{c}}$ variation can be achieved by increasing the thermoelectric figure of merit.

It is to be noted that a useful relationship can be derived from equation (4) by considering two special cases: open circuit $\left(R_{\mathrm{L}}=\infty\right)$ and short circuit $\left(R_{\mathrm{L}}=0\right)$. If the external resistance is switched between these two extreme values, equation (4) can be simplified to

$$
\frac{\Delta T_{\mathrm{o}}}{\Delta T_{\mathrm{s}}}=1+Z \bar{T}
$$

where $\Delta T_{\mathrm{o}}$ and $\Delta T_{\mathrm{S}}$ are temperature differences under open circuit and short circuit, respectively. $\bar{T}=\left(T_{\mathrm{A}}+T_{\mathrm{B}}\right) / 2$ is the mean temperature over the module. Equation (5) has 


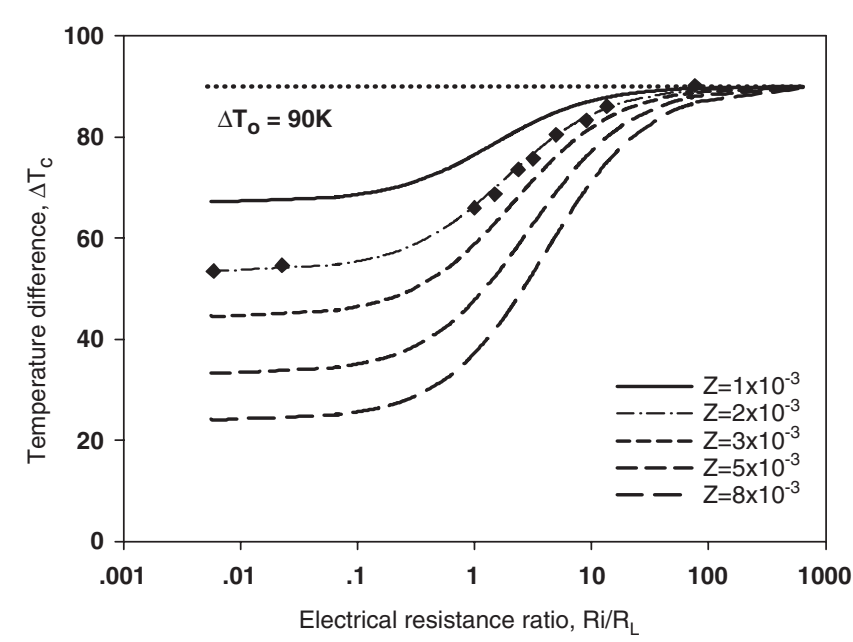

Figure 2. Temperature difference between two thermal bodies as a function of the ratio of $R_{\mathrm{L}} / R_{\mathrm{i}}$ for different values of thermoelectric figure of merit. The solid dots represent the experimental result and dashed lines are calculated ones.

been reported as a measurement principle for developing a new technique for direct evaluation of the dimensionless thermoelectric figure of merit [10]. A distinctive advantage of this technique is that it facilitates the $Z T$ evaluation under a large temperature difference [11].

In conclusion, equation (4) describes a general relationship between heat flux and temperature difference in a thermoelectric circuit shown in figure 1. This relationship may be viewed as a 'modified Fourier law' in which the thermal conductance of thermoelectric materials can be controlled by the electrical resistance of an external resistor connected to it. As a result, a thermoelectric material or module can be employed as a variable thermal resistor for applications where the heat flux or temperature difference between two thermal bodies need to be altered conveniently. The extent of variation in thermal resistance is mainly dependent on the thermoelectric figure of merit. Currently, a change in heat flux (or temperature difference) of up to a factor of 2 can be obtained using commercial modules. To increase the variation range, it is necessary to improve the thermoelectric figure of merit.

\section{Reference}

[1] Rowe D M and Bhandari C M 1983 Modern Thermoelectrics (London: Holt, Rinehart and Winston)

[2] Goldsmid H J 1986 Electronic Refrigeration (London: Pion)

[3] Pollock D D 1991 Thermocouples: Theory and Properties (London: CRC Press)

[4] Wood R A, Han C J and Kruse P W 1992 Proc. IEEE Solid State Sensor and Actuator Workshop (Hilton Head Island, SC) $\mathrm{p} 132$

[5] Herwaarden W V and Sarro P M 1986 Sensors Actuators 10321

[6] Sher E M 1987 Proc. 1st European Conf. on Thermoelectrics (Cardiff, UK) ed D M Rowe (London: Peter Peregrinus) p 277

[7] Min G and Rowe D M 2007 Semicond. Sci. Technol. 22880

[8] Szekely V, Nagy A, Torok S, Hajas G and Rencz M 2001 Microelectron. J. 31811

[9] Min G 2005 CRC Handbook of Thermoelectrics: Macro to Nano ed D M Rowe (London: CRC Press) 11-1

[10] Min G and Rowe D M 2001 J. Meas. Sci. Technol. 121261

[11] Min G, Rowe D M and Kontostavlakis K 2001 J. Phys. D: Appl. Phys. 371301 